\title{
Rhinoplasty Education Using a Standardized Patient Encounter
}

\author{
Eric J. Wright, Rohit K. Khosla, Lori Howell, Gordon K. Lee \\ Division of Plastic and Reconstructive Surgery, Stanford University, Palo Alto, CA, USA
}

Background Comprehensive aesthetic surgery training continues to be a challenge for residency programs. Our residency program developed a rhinoplasty-based objective structured clinical examination (OSCE) based upon validated methods as part of the residency education curriculum. We report our experience with the rhinoplasty-based OSCE and offer guidance to its incorporation within residency programs.

Methods The encounter involved resident evaluation and operative planning for a standardized patient desiring a rhinoplasty procedure. Validated OSCE methods currently used at our medical school were implemented. Residents were evaluated on appropriate history taking, physical examination, and explanation to the patient of treatment options. Examination results were evaluated using analysis of variance (statistical significance $\mathrm{P}<0.05$ ).

Results Twelve residents completed the rhinoplasty OSCE. Medical knowledge assessment showed increasing performance with clinical year, 50\% versus $84 \%$ for postgraduate year 3 and 6 , respectively $(P<0.005)$. Systems-based practice scores showed that all residents incorrectly submitted forms for billing and operative scheduling. All residents confirmed that the OSCE realistically represents an actual patient encounter. All faculty confirmed the utility of evaluating resident performance during the OSCE as a useful assessment tool for determining the Next Accreditation System Milestone level.

Conclusions Aesthetic surgery training for residents will require innovative methods for education. Our examination showed a program-educational weakness in billing/coding, an area that will be improved upon by topic-specific lectures. A thoroughly developed OSCE can provide a realistic educational opportunity to improve residents' performance on the nonoperative aspects of rhinoplasty and should be considered as an adjunct to resident education.

Keywords Rhinoplasty / Educational measurement / Internship and residency
Correspondence: Gordon K. Lee Division of Plastic and Reconstructive Surgery, Stanford University, 770 Welch Rd, \#400, Palo Alto, CA 94304-5715, USA

Tel: +1-650-723-5824

Fax: +1-650-725-6605

E-mail: glee@stanford.edu
This article was oral presented at The Rhinoplasty Society 19th Annual Meeting on April 24, 2014 in San Francisco, CA, USA

No potential conflict of interest relevant to this article was reported.

\section{INTRODUCTION}

The challenges and possible solutions related to adequately training residents in aesthetic surgery have been debated for decades. Numerous solutions have been discussed based upon surveys of current training programs [1,2]. In the 2011 survey by Oni et al. [1], over $50 \%$ of program directors did not feel confident in the residents' ability to perform a rhinoplasty and $60 \%$ of residents desired more training in rhinoplasty procedures. There are several reasons for this disparity. Despite the complexity of evaluating rhinoplasty patients and performing the procedure, the current Accreditation Council for Graduate Medical 
Education (ACGME) requirements for graduating plastic surgery residents are 6 rhinoplasty cases. Aesthetic rhinoplasty cases are also infrequently performed in academic university hospitals. Unless the residency program has a resident cosmetic clinic that routinely performs rhinoplasty cases, exposure to rhinoplasty cases occurs in the private-practice setting, which is more of an observational experience. Additionally, exposure to rhinoplasty cases typically occurs in the senior postgraduate years [3].

The paucity of early exposure and training in rhinoplasty can create challenges for residency program curricula. Evaluation of plastic surgery residents has recently transitioned to a competency-based evaluation system. The ACGME Next Accreditation System (NAS) has restructured the resident and program evaluation process from the 6 core competencies developed in 1999 [4]. As part of NAS, The Plastic Surgery Milestone Project was formed [5]. This project created milestones designed for an outcomes-based assessment of residents as they progress through the training program by designating a level of 1 through 5 based upon their current capabilities and attributes [5]. These milestones were implemented within plastic surgery programs in July of 2014. The implementation of the milestones within the educational curriculum and the mechanisms and tools used to assess the resident milestone level have been left to the individual resident programs, with no consensus of the best method to date. Without adequate exposure to rhinoplasty cases, it is difficult to designate a milestone level for a resident within this area.

With the need for objective evaluation tools, our institution has incorporated within the residency program the use of objective structured clinical examinations (OSCEs) [6,7]. The utility of OSCEs as a valid and reliable assessment tool, including assessment of subsets of the core competencies, has been well established within multiple medical and surgical fields [8-12].

In this manuscript, we describe the implementation of the first published comprehensive cosmetic standardized patient exam, a rhinoplasty OSCE that includes evaluation components of all 6 ACGME core competencies. We believe the benefits of OSCEs include the education of residents in cosmetic surgery and as an evaluation tool used to assess the resident Milestone Project level. Our OSCE experience can be a used as a guideline for other training programs in the implementation of OSCEs. Institutional Review Board exemption was obtained for this manuscript.

\section{METHODS}

Residents in years 3-6 were evaluated on appropriate history taking, physical examination, and treatment-option explanation to the patient. Validated OSCE methods currently used at our medical school were implemented. Components of the OSCE were created to allow for assessment of the ACGME 6 core competencies required for resident evaluation.

\section{Clinical scenario}

The encounter involved the resident evaluation and operative planning for a standardized patient (SP) desiring a rhinoplasty procedure: a 30-year-old woman presenting to the clinic concerned about the appearance of her nose. Residents completed examinations, performed literature reviews to support certain procedures, and submitted billing and operative scheduling

\section{Fig. 1. A standardized patient photo for surgical planning}
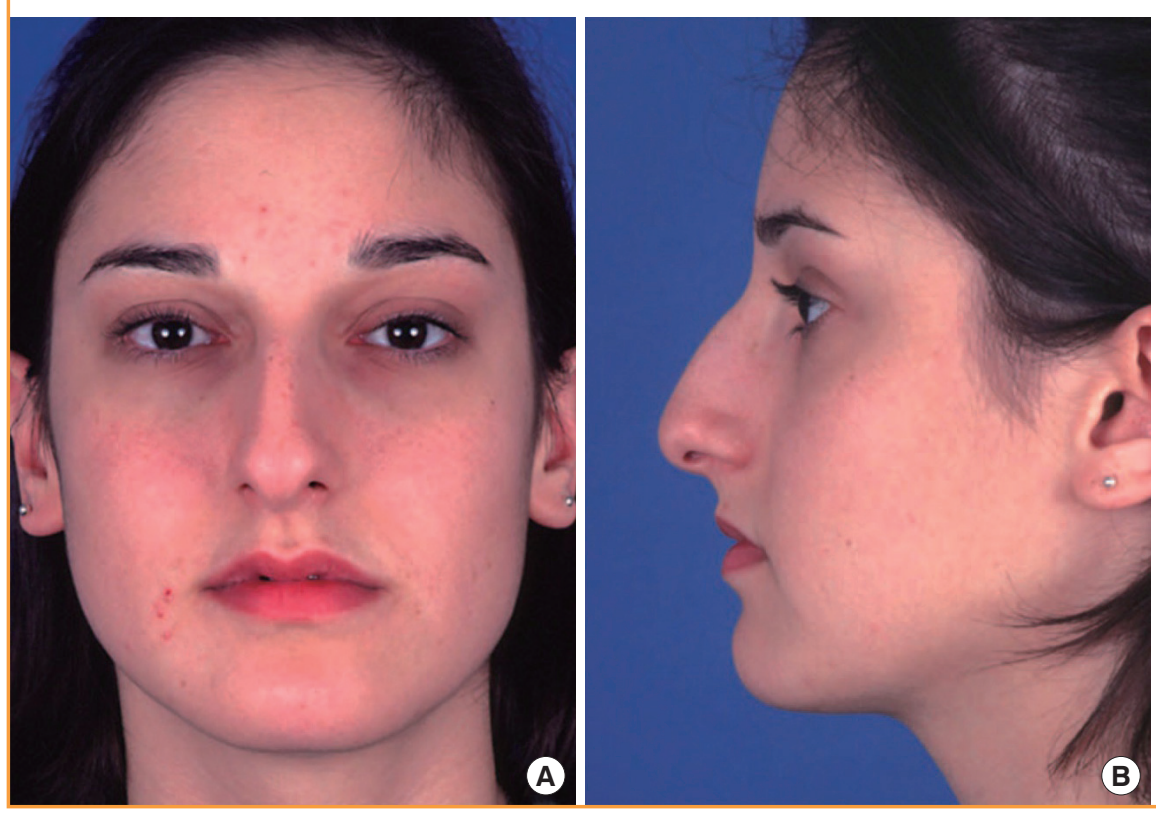

(A) Frontal view of a standardized patient provided to all residents for developing an analysis and surgical plan. (B) Lateral view of the standardized patient. (C) Worm's eye view of the standardized patient.

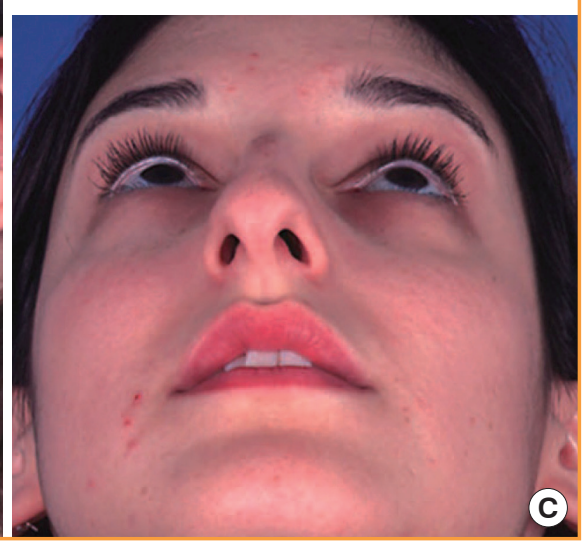


forms. SPs completed assessments of the professionalism and communication performance. Residents were given a survey following review of the examination with attending faculty. Results were stratified per resident year and evaluated using analysis of variance $(\mathrm{P}<0.05)$.

\section{Standardized patient encounter}

Two standardized female patient actors were trained per protocol. In order to standardize the operative planning for all residents, three photos were provided of a patient (Fig. 1). Residents were expected to determine if the SP had any nasal obstruction symptoms and what the patient-specific aesthetic concerns were. The SP provided a history of trauma and displayed a positive Cottle sign on examination. After the physical exam, residents were to discuss the appropriate management of the condition and provide the appropriate education regarding future visits or procedures. Twenty minutes were provided to complete this video-recorded encounter (Fig. 2).

\section{Post-encounter exercises and resident evaluation}

Following the SP encounter, residents performed several additional exams in order to complete the OSCE allowing for assessment of the ACGME 6 core competencies. For medical knowl-

\section{Fig. 2. Interaction between the resident and standardized patient}

The resident examination is video recorded, allowing for review by attending faculty.

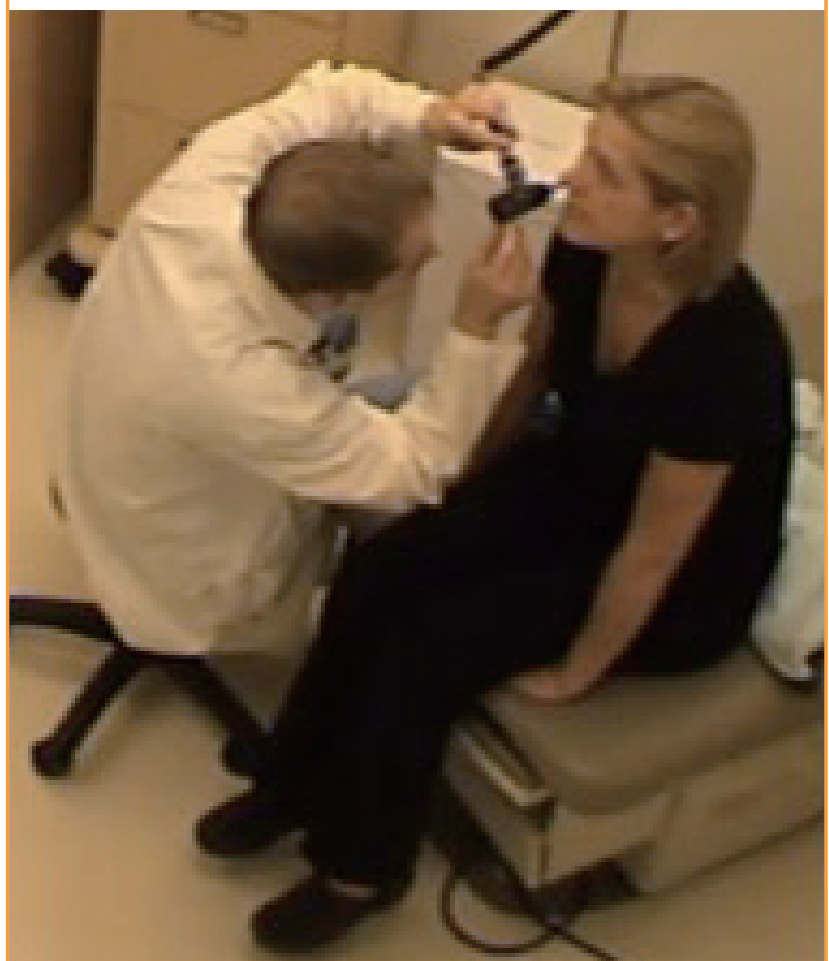

edge: a written explanation of the patient physical exam findings and a written step-by-step operative plan were graded (Table 1). A multiple-choice (in-service style) exam was also administered. For systems-based practice: current procedural terminology (CPT) coding, international classification of diseases (ICD)-9, and billing forms were completed. A PubMed search was performed to reference support "for or against the use of spread grafts in the treatment of nasal airway obstruction," allowing for problem-based learning and improvement evaluation. The remaining 3 core competencies (patient care, professionalism, and interpersonal communication) were evaluated based upon SP evaluation and checklists. Two attendings independently reviewed the video-recorded SP encounters and written-exam results. Individual resident, faculty, and program review of results was performed to look for areas of deficiency to allow for improvement of education within the residency curriculum.

\section{RESULTS}

Twelve residents completed the rhinoplasty OSCE. For the

$$
\text { Table 1. Example written patient physical exam }
$$

Rhinoplasty OSCE patient exam
Based upon patient exam and review of provided pictures, please answer the
following questions about this encounter.
1. What are the major deformities of this patient's nose? (briefly describe your
exam findings) (5 pts)
Upper 1/3:
Middle 1/3:
Lower 1/3:
2. Which side has internal nasal valve collapse? (1 pt)
3. If your patient has internal nasal valve collapse, how would you repair it? (1 pt)
4. If your patient has external nasal valve collapse, how would you repair it? (1 pt)
5. Where will you obtain the cartilage graft? How will you gain access to this
graft? ( 2 pts)
6. What is your overall operative plan? (7 pts)

Clinical knowledge is tested by various methods, including written examination. OSCE, objective structured clinical examination; pts, points.

\section{Fig. 3. Resident written examination scores}

PGY, postgraduate year.

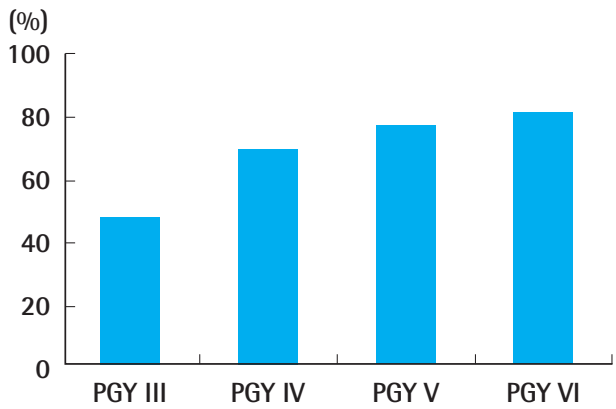


Table 2. Itemized rhinoplasty OSCE expenses

\begin{tabular}{|c|c|c|c|c|c|c|c|c|}
\hline Personnel & No. & Hours & $\begin{array}{c}\text { Hourly rate } \\
\text { (\$) }\end{array}$ & $\begin{array}{c}\text { Total wages } \\
(\$)\end{array}$ & $\begin{array}{l}\text { No. of } \\
\text { permits }\end{array}$ & $\begin{array}{c}\text { Parking cost } \\
(\$)\end{array}$ & $\begin{array}{l}\text { Parking total } \\
(\$)\end{array}$ & $\begin{array}{c}\text { Daily total } \\
(\$)\end{array}$ \\
\hline \multicolumn{9}{|l|}{ Preparation } \\
\hline Coordinator & 1 & 10 & 25 & 250 & 0 & & & 250 \\
\hline \multicolumn{9}{|l|}{ Training day 1} \\
\hline Coordinator & 1 & 2 & 25 & 50 & 1 & 4 & 4 & 54 \\
\hline $\mathrm{SP}$ & 2 & 2 & 15 & 60 & 2 & 4 & 8 & 68 \\
\hline \multicolumn{9}{|l|}{ Training day 2} \\
\hline Coordinator & 1 & 2 & 25 & 50 & 1 & 4 & 4 & 54 \\
\hline $\mathrm{SP}$ & 2 & 2 & 15 & 60 & 2 & 4 & 8 & 68 \\
\hline Training total & & & & & & & & 494 \\
\hline \multicolumn{9}{|l|}{ Exercise } \\
\hline $\mathrm{SP}$ & 2 & 4 & 15 & 120 & 2 & 4 & 8 & 128 \\
\hline Proctor & 1 & 4 & 15 & 60 & 1 & 4 & 4 & 64 \\
\hline Coordinator & 1 & 4 & 25 & 100 & 1 & 4 & 4 & 104 \\
\hline Total for OSCE & & & & & & & & 296 \\
\hline Grand total & & & & & & & & 790 \\
\hline
\end{tabular}

written exam, assessment of both medical knowledge and the patient examination showed increasing performance with clinical year, $50 \%$ vs. $84 \%$ for postgraduate year (PGY) 3 and 6, respectively (Fig. 3). Systems-based practice scores found that all residents incorrectly submitted forms for billing and operative scheduling, lacking the distinction between reconstructive versus cosmetic procedures. SP evaluations varied per resident for areas of interpersonal and communication skills, professionalism, and patient care, with higher scores not correlating to PGY year. This OSCE was used solely as an educational assessment tool and learning opportunity.

Only the PGY 6 residents had performed a cosmetic rhinoplasty, with the PGY 5 residents having exposure to cleft-rhinoplasty procedures during a pediatric rotation. All residents confirmed that the OSCE realistically represents an actual patient encounter and that this was an educational experience.

The overall total cost for the OSCE was $\$ 790$ or $\$ 66 /$ resident (Table 2). Administration of the OSCE required the use of a coordinator who was responsible for training the two SPs and review of the rhinoplasty scenario that had been created to ensure it met all OSCE standards required at our institution's medical school.

\section{DISCUSSION}

There is no greater area of debate within plastic surgery resident education than how to improve the exposure and competency of aesthetic surgery training. Having resident cosmetic clinics or pursuing aesthetic surgery fellowships have both been discussed as possible solutions. However, not all programs are able to accommodate the resident clinics. In addition, residents should be competent to perform aesthetic procedures without the need for additional training. Our residency program has begun to explore the incorporation of different OSCE scenarios within the yearly curriculum. OSCEs have been a proven and validated method for medical student and resident education. Numerous medical and surgical subspecialties have written about their experiences with OSCEs in training situations and have confirmed their benefit as a validated resident-education tool $[8,12,13]$. Additionally, OSCEs have been used to evaluate the individual core competencies of surgical residents based upon their interactions with a standardized patient $[7,14]$. Even with plastic surgery, OSCEs have been shown to be beneficial in teaching residents appropriate patient selection in aesthetic surgery [15]. This OSCE represents a realistic patient encounter that allows for assessment of the trainee's competency to interact with and evaluate a cosmetic patient. This allows for a controlled environment where residents are able to freely examine and independently discuss the surgical plans, something that does not always happen when observing in private practice.

Rhinoplasty consistently ranks as one of the most difficult and demanding procedures to learn. Determining the correct patient to offer a procedure and having a detailed surgical plan based upon thorough facial analysis are key components for the success of the operation, all developed before ever entering the operating room. Learning the intricacies of nasal analysis is a topic that takes significant preparation and exposure [16]. Our goal was to assess and teach the fundamentals of an initial rhinoplasty patient clinic encounter. Studies have noted that resident-run clinics are the best way to learn, as opposed to observation [17]. Ideally, residents would complete OSCEs before evaluating actual patients. Given that resident clinics do not always have a 
high volume of rhinoplasty cases, it would allow for residents to "make the most" of their rhinoplasty patients.

Upon review of our rhinoplasty OSCE, all residents and faculty confirmed this was an educational exercise. Though the medical knowledge component of the exam found scores significantly greater for PGY 6 level residents compared to more junior residents, the remaining core competency scores were not grouped by postgraduate year. As discussed more in detail later, the milestone levels are an individual resident assessment, and are not designated to a specific year of training. It has been noted that the more subjective competencies such as professionalism and communication skills are reflective of the resident's inherent characteristics and would not necessarily become more advanced each year [7]. The results of the OSCE also allow for an evaluation of the educational curriculum. Residents inappropriately coded their chosen procedures, showing there was some confusion on this topic. One discrepancy is in distinguishing cosmetic versus reconstructive procedures. All residents expressed interest in earlier and more extensive exposure to rhinoplasty patients.

As discussed in previous OSCE experiences, the initial scenario creation requires the most investment in time and cost $[6,18]$. The overall total cost for the OSCE was $\$ 790$. Per resident, the cost was $\$ 66$, which has decreased from our other OSCEs. The initial preparation and utilization of the trainer accounted for over 50\% of the total cost. Once the format and OSCE design requirements have been learned, any scenario can be created without a significant time requirement from the trainer. We have learned from our previous OSCE administration experience and continue to decrease the overall cost.

Another benefit we perceive with the OSCE format is the longitudinal and qualitative ability to assess residents as they progress throughout the training program. We have found that the resident OSCE performance is a reliable tool to allow for assessment of the ACGME requirements. Implementation of the ACGME's NAS and Plastic Surgery Milestones in 2014 has developed guidelines for evaluation of resident performance that residents will obtain independent of their year of training. OSCEs serve as a comprehensive outcomes-based tool that incorporates all 6 core competencies for demonstrating the specific milestone levels. The milestone evaluation emphasizes resident assessment based upon observable behaviors using a variety of tools. By nature of having video-recorded resident-patient encounters, an OSCE represents an impartial, reviewable, and longitudinal assessment tool by evaluating the resident's repeat performance as they advance in resident training.

OSCEs do have their limitations. There is the continued issue with the cost of conducting an OSCE. Faculty review and feed- back for the residents takes a significant time commitment. However, since all encounters are video recorded, this can occur over the subsequent weeks with still meaningful critiques. Though we continue to decrease our financial cost of the OSCEs, it will still require funding from some source. One of the more challenging limitations specific to aesthetic surgery is the innate variation in the proposed assessment and operative plan. Though our OSCE resident evaluation was based upon a "correct analysis and surgical plan" proposed by two attendings, the surgical plans proposed by other programs' attendings may vary. Also, the rhinoplasty OSCE only represents a single patient encounter, as there are numerous possible rhinoplasty scenarios.

Comprehensive aesthetic surgery training continues to be a challenge for residency programs. The education for residents must include more than the operative technique alone. Our residency program developed a rhinoplasty-based OSCE based upon validated methods as part of the residency-education curriculum. The use of OSCEs in resident training is not a novel idea, but as we search for ideal assessment tools, it does have its place within residency training. No single assessment can provide all the information needed for milestone evaluation. Aesthetic surgery training for residents will require innovative methods for education. A thoroughly developed OSCE can provide a realistic educational opportunity to improve residents' performance on the non-operative aspect of rhinoplasty and should be considered as an adjunct to resident education.

\section{REFERENCES}

1. Oni G, Ahmad J, Zins JE, et al. Cosmetic surgery training in plastic surgery residency programs in the United States: how have we progressed in the last three years? Aesthet Surg J 2011;31:445-55.

2. Morrison CM, Rotemberg SC, Moreira-Gonzalez A, et al. A survey of cosmetic surgery training in plastic surgery programs in the United States. Plast Reconstr Surg 2008;122: 1570-8.

3. Accreditation Council for Graduate Medical Education Accreditation Council for Graduate Medical Education. ACGME Program and Institutional Accreditation Plastic Surgery [Internet]. Chicago, IL: Accreditation Council for Graduate Medical Education Accreditation Council for Graduate Medical Education; c2016 [cited 2015 Dec 17]. Available from: https://www.acgme.org/acgmeweb/tabid/145/ProgramandInstitutionalAccreditation/SurgicalSpecialties/PlasticSurgery.aspx.

4. Nasca TJ, Philibert I, Brigham T, et al. The next GME accreditation system: rationale and benefits. N Engl J Med 2012; 
366:1051-6.

5. The Plastic Surgery Milestone Project [Internet]. Chicago, IL: The Accreditation Council for Graduate Medical Education and The American Board of Plastic Surgery, Inc.; c2013 [cited 2015 Dec 17]. Available from: https://www.acgme. org/acgmeweb/Portals/0/PDFs/Milestones/PlasticSurgeryMilestones.pdf.

6. Wright EJ, Khosla RK, Howell L, et al. Cleft Lip standardized patient examinations: the role in plastic surgery resident education. Cleft Palate Craniofac J 2015 Dec 31 [Epub]. http:// dx.doi.org/10.1597/15-121.

7. Davis D, Lee G. The use of standardized patients in the plastic surgery residency curriculum: teaching core competencies with objective structured clinical examinations. Plast Reconstr Surg 2011;128:291-8.

8. Griesser MJ, Beran MC, Flanigan DC, et al. Implementation of an objective structured clinical exam (OSCE) into orthopedic surgery residency training. J Surg Educ 2012;69:180-9.

9. Franzese C. When to cut? Using an objective structured clinical examination to evaluate surgical decision-making. Laryngoscope 2007; 117:1938-42.

10. Hochberg MS, Kalet A, Zabar S, et al. Can professionalism be taught? Encouraging evidence. Am J Surg 2010;199:8693.

11. Schwartz RW, Witzke DB, Donnelly MB, et al. Assessing residents' clinical performance: cumulative results of a fouryear study with the Objective Structured Clinical Examination. Surgery 1998;124:307-12.

12. Sloan DA, Donnelly MB, Schwartz RW, et al. The Objective Structured Clinical Examination. The new gold standard for evaluating postgraduate clinical performance. Ann Surg 1995; 222:735-42.

13. Wolff MJ, Balzora S, Poles M, et al. Objective structured clinical examination as a novel tool in inflammatory bowel disease fellowship education. Inflamm Bowel Dis 2015;21:75965.

14. Hassett JM, Zinnerstrom K, Nawotniak RH, et al. Utilization of standardized patients to evaluate clinical and interpersonal skills of surgical residents. Surgery 2006;140:633-8.

15. Rinker B, Donnelly M, Vasconez HC. Teaching patient selection in aesthetic surgery: use of the standardized patient. Ann Plast Surg 2008;61:127-31.

16. Byrd HS, Hobar PC. Rhinoplasty: a practical guide for surgical planning. Plast Reconstr Surg 1993;91:642-54.

17. Linder SA, Mele JA 3rd, Capozzi A. Teaching aesthetic surgery at the resident level. Aesthetic Plast Surg 1996;20:351-4.

18. Son J, Zeidler KR, Echo A, et al. Teaching core competencies of reconstructive microsurgery with the use of standardized patients. Ann Plast Surg 2013;70:476-81. 